\title{
A COMB-DRIVE ACTUATOR ENABLED DYNAMICALLY TUNABLE TERAHERTZ METAMATERIAL
}

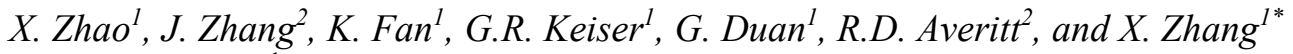 \\ ${ }^{1}$ Boston University, Boston, Massachusetts, USA \\ ${ }^{2}$ University of California, San Diego, La Jolla, California, USA
}

\begin{abstract}
This paper reports our recent progress on a dynamically tunable terahertz metamaterial based on broadside-coupled split ring resonators (BC-SRRs) and a comb-drive actuator. In the structure, the coupling between two arrays of SRR is modulated with the lateral displacement exerted by the comb-drive actuator. In the spectrum of BC-SRRs, two resonant modes can be observed, i.e. symmetric mode and anti-symmetric mode, due to the strong inductive and capacitive coupling. Over $20 \mu \mathrm{m}$ lateral displacement, the symmetric mode blueshifts $\sim 60 \mathrm{GHz}$ and the anti-symmetric mode redshifts $\sim 50 \mathrm{GHz}$. The amplitude of the transmission at 1.03 $\mathrm{THz}$ is modulated by $69 \%$. We can realize a large tuning range with structure optimization, thereby enabling the applications including $\mathrm{THz}$ modulators and chemical sensing.
\end{abstract}

\section{INTRODUCTION}

Over the past decade, metamaterials (MMs) have been proved to be a scheme to engineer the electromagnetic response of materials through resonance excitations [1]. They have many remarkable applications, such as negative refractive index [2], super lensing [3], perfect absorption [4], and cloaking [5], in information processing [6], chemical and biology sensing [7], and security [8,9].

In order to enhance the functionalities of MMs, a number of approaches have been employed to make the MMs reconfigurable. These include electrical gating [10], photo-excitation [11], thermal tuning [12], modulation of near-field coupling [13] and structural and mechanical reconfiguration [14]. Among these methods, tuning the near field coupling is of interest because of its high efficiency, stability and ease of controlling [15]. One coupled MM structures is broadside-coupled split ring resonator (BC-SRR), including two split ring resonators (SRRs) arrays are stacked vertically and rotated $180^{\circ}$ to each other. Recent study shows that the lateral displacement between the two SRR layers will tune the MM resonance frequency [13].

Micromachined electrostatic actuators provide an intuitive path to realize the functional broadside-coupled tunable devices. We demonstrated implantation of the real-time tunable metamaterial based on the BC-SRRs and comb-drive actuators in [16].

In this paper, we report our recent process of the BC-SRRs based metamaterial at $\mathrm{THz}$ frequencies. The coupling between the SRRs is optimized with new geometry design and the fabrication process is improved to achieve good alignment in the flip-chip bonding. With the new mechanical structure design, the yield of the functional device is increased as well. With this improvement, the tunability is improved significantly compare to our previous work [16]. The first resonant mode redshifts $60 \mathrm{GHz}$ and the second mode redshifts $50 \mathrm{GHz}$ with $20 \mu \mathrm{m}$ lateral displacement achieved by the comb-drive actuator. Meanwhile, the amplitude at $1.03 \mathrm{THz}$ is modulated by $69 \%$.

\section{DESIGN AND FABRICATION}

The illustration of the designed tunable MMs is shown in Figure 1. The MMs are composed with two layers of SRRs separated by $20 \mu \mathrm{m}$ air gap. One layer is on $10 \mu \mathrm{m}$ thick silicon with electrostatic comb-drive actuators, while the other is fixed on 500 $\mathrm{nm}$ thick $\mathrm{SiN}_{\mathrm{x}}$ thin film. The unit-cell of the MMs with different $\mathrm{x}$-axis displacement is shown in Figure1 (b). The rings are rotated $180^{\circ}$ to each other, forming a broadside coupled configuration. Since the two layers of MMs are closed to each other, the near-field inductive and capacitive interactions between them are strong. The relative shift along $\mathrm{x}$-axis between the arrays, controlled by the applied voltage, can change the coupling between the SRRs. Thus, it modulated the frequency response of the MM.

In this device, the geometries of the two SRRs in each coupled pair are not identical to compensate for the effect of the different substrates. We determine the parameters, as listed in Table 1, using numerical simulation in order to match the resonant frequency of the uncoupled SRRs. The frequency match can improve the coupling efficiency between the SRRs and tunability comparing to the unmatched condition [16]. The simulated transmission spectrum of SRR1 and SRR2 are shown in Figure 2. The dip in the each spectrum corresponds to the LC resonant frequency of the SRR, which is determined by the self-inductance and capacitance of each ring. When the two rings are stacked together, the mutual inductance and capacitance will show up and lead to the frequency split in the spectrum [17], as shown with the red curve in Figure 2.

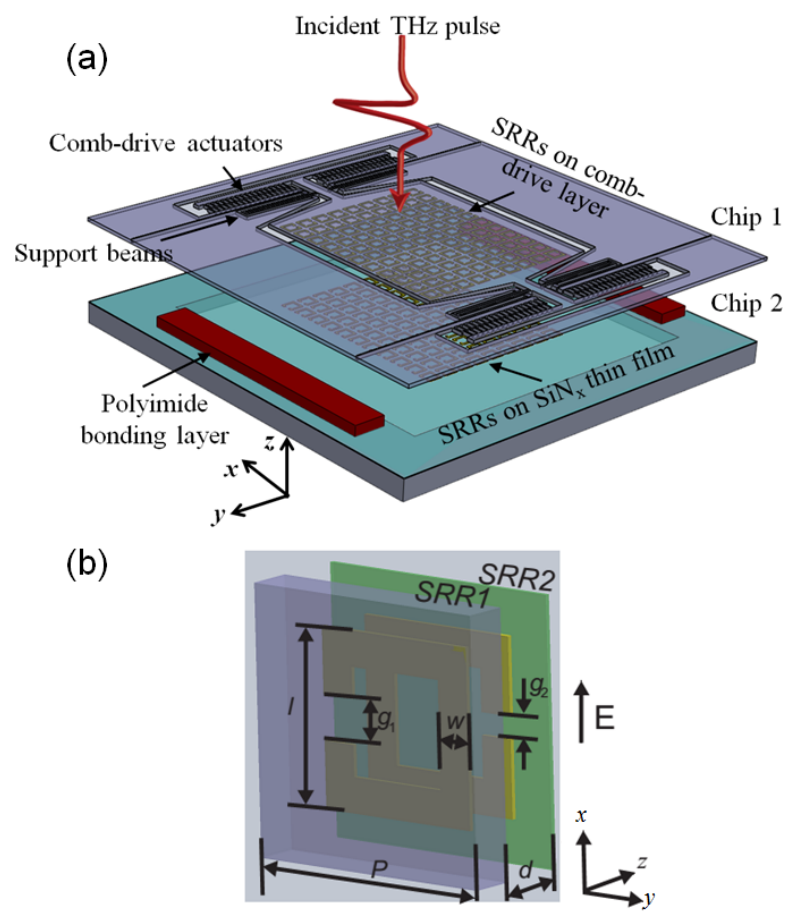

Figure 1: Illustration of the broad-side coupled tunable metamaterials: (a) the exploded diagram of the tunable metamaterials, in which two chips were bonded together with polyimide spacer; (b) and (c) the unit-cell of the metamaterials with different $x$-axis shift.

Solid-State Sensors, Actuators and Microsystems Workshop Hilton Head Island, South Carolina, June 5-9, 2016 
Table 1: The dimensions of SRRs (unit in $\mu \mathrm{m}$ )

\begin{tabular}{|c|c|c|c|c|}
\hline & $\begin{array}{c}\text { Periodicity } \\
(P)\end{array}$ & $\begin{array}{c}\text { Side-width } \\
(l)\end{array}$ & $\begin{array}{c}\text { Linewdith } \\
(w)\end{array}$ & $\begin{array}{c}\text { Gap } \\
(g)\end{array}$ \\
\hline SRR1 & 58 & 40 & 11 & 16 \\
\hline SRR2 & 58 & 40 & 11 & 2 \\
\hline
\end{tabular}

The tunable metamaterial was fabricated with micromachining processes as shown in Figure 3, which include two wafer processes and flip chip bonding. An SOI wafer with a $10 \mu \mathrm{m}$ thick device layer and $2 \mu \mathrm{m}$ buried oxide layer was used as the first wafer. Both sides of the wafer were coated by $400 \mathrm{~nm}$ thick low-stress $\mathrm{SiN}_{\mathrm{x}}$ films grown by LPCVD. With subsequent photolithography and reactive ion etching (RIE), the $\mathrm{SiN}_{\mathrm{x}}$ film on the top side was patterned [Figure 3 (a)]. Then, the electrodes were deposited on the exposed silicon using e-beam evaporation of $400 \mathrm{~nm} \mathrm{Al}$. The ohmic contact was achieved by annealing at $450{ }^{\circ} \mathrm{C}$ in $\mathrm{H}_{2} / \mathrm{N}_{2}$ ambient. The electrodes were coated with a $150 \mathrm{~nm}$ gold thin film after annealing [Figure 3 (b)]. Subsequently, $150 \mathrm{~nm}$ thick gold SRRs were patterned via a lift-off process and the device layer was etched using deep reactive ion etching (DRIE) to form the comb-drive actuators and supporting beams [Figure 3 (c) and (d)]. A $600 \mathrm{~nm}$ thick $\mathrm{SiO}_{2}$ passivation layer was deposited on the front side with PECVD in order to protect the patterned device layer in the following steps [Figure $3(\mathrm{e})$ ]. Next, backside alignment and photolithography using AZ9260 (MicroChemicals, Inc.) was performed and the $\mathrm{SiN}_{\mathrm{x}}$ film was etched with RIE, followed by etching through the Si substrate using DRIE [Figure 3 (f) and (g)]. The last step [Figure $3(\mathrm{~h})$ ] was to etch the passivation layer and buried oxide layer with Silox Vapoxy III etchant (Transene, Inc.) to release the movable structure. The second wafer, a single crystal $<100>$ silicon wafer, was coated with low-stress $\mathrm{SiN}_{\mathrm{x}}$ film on both sides using LPCVD. Then, $150 \mathrm{~nm}$ thick gold SRRs were patterned on the top side with lift-off process [Figure 3 (i)]. Then, the silicon wafer was etched through with $\mathrm{KOH}$ wet etching from the window opened on the backside $\mathrm{SiN}_{\mathrm{x}}$ film [Figure 3 (j)]. After the wafer-level fabrication, the two wafers were diced into chips. For clarity and conciseness, we name the chips from the SOI wafer as chip 1 and the chips from the silicon wafer as

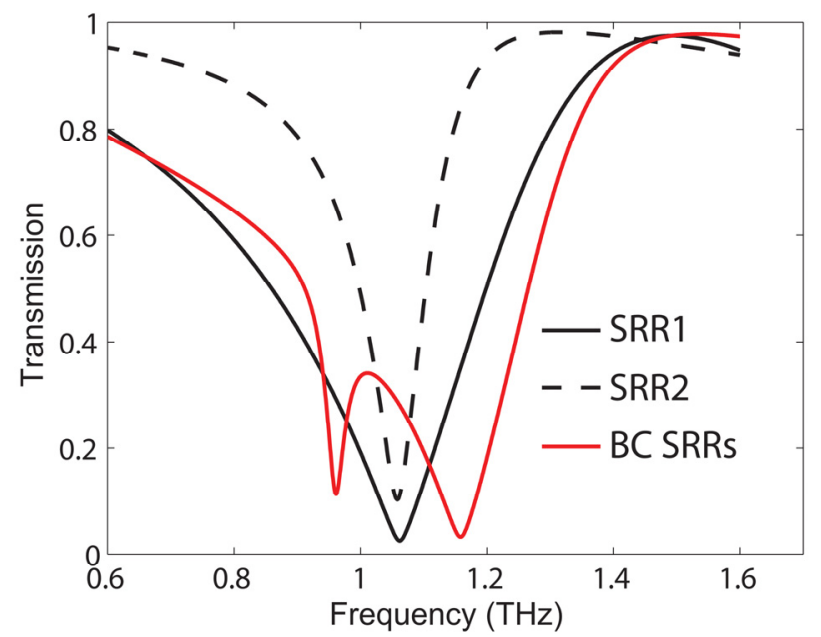

Figure 2: The simulated response of the SRR1, SRR2 and $B C-S R R s$ with the geometries listed in Table 1. The resonant frequencies of the SRR1 and SRR2 are matched. The vertical distance between the SRRs is $20 \mu \mathrm{m}$ in the BC-SRRs. Frequency splitting shows up in the BC-SRRs. chip 2. Polyimide bonding pads were patterned on chip 2 via photolithography of the photosensitive polyimide (HD8820, HD Microsystems). Finally, chip 1 and chip 2 were bonded together using a flip chip bonder (FC150, Suss MicroTec AG) under $200 \mathrm{~g}$ force and $175{ }^{\circ} \mathrm{C}$ temperature. The bonded chips were connected with a printed circuit board via bonding wire to apply the voltage.

The fabricated device is shown in Figure 4 (a). Details of the chip 1 are shown in Figure 4 (b) and (c). The microscope images of the bonded device [Figure 4 (d) and (e)] show that the SRRs are well-aligned in $\mathrm{y}$ direction and the misalignment in the $\mathrm{x}$ direction can be controlled with the comb-drive actuated lateral displacement.

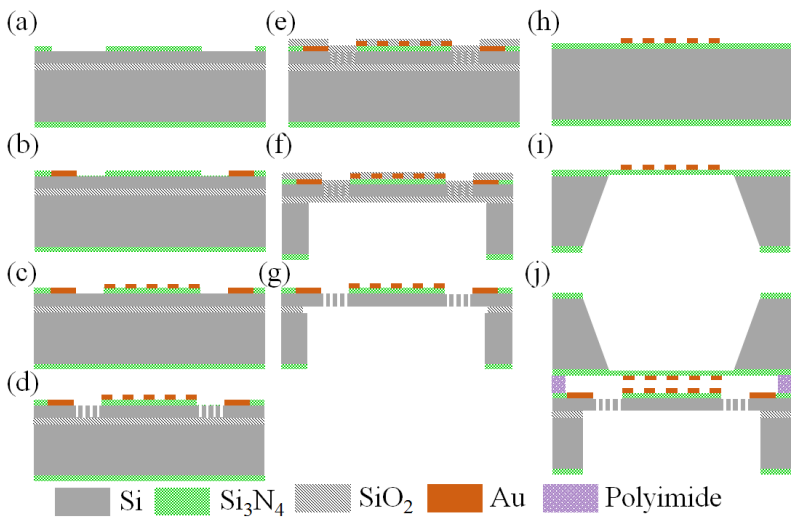

Figure 3: Fabrication process of the broad-side coupled tunable metamaterials: (a)-(h) the first (SOI) wafer process; (i)-(j) the second (silicon) wafer process; $(k)$ the flip-chip bonding. The bonded chips were connected with a printed circuit board via bonding wire to apply the voltage

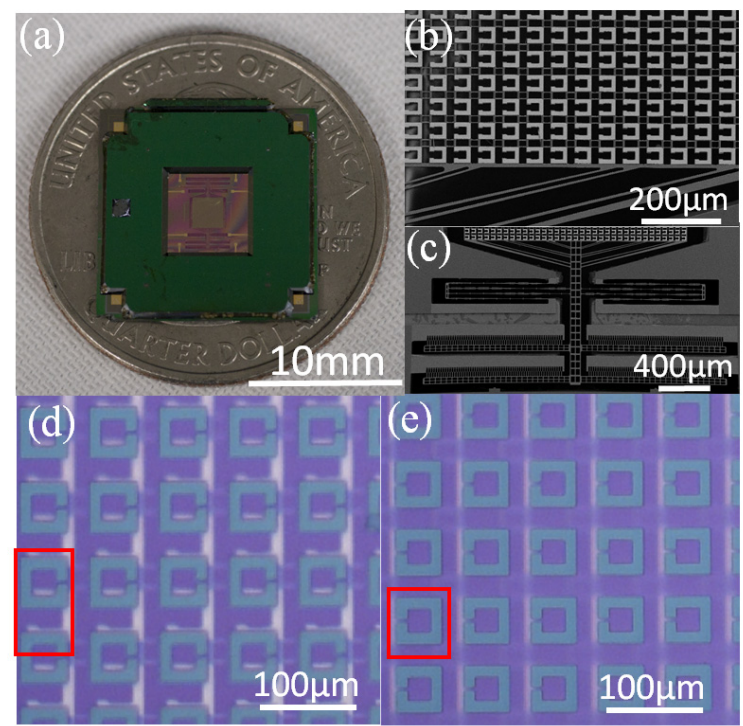

Figure 4: Fabricated device.(a) The flip-chip bonded tunable metamaterial; (b) and (c) SEM images of the comb-drive chip;(d) the SRRs with O $\mu$ m lateral displacement, where the SRRs are aligned; (e) the SRRs with $20 \mu \mathrm{m}$ relative lateral displacement, where the SRRs are misaligned.

\section{EXPERIMENT AND RESULTS}

The electromagnetic response of the real-time tunable metamaterial was characterized by $\mathrm{THz}$ time domain spectroscopy 
(THz-TDS), as shown in Figure 5. A $1 \mathrm{kHz}$ Ti:sapphire femtosecond laser producing $1.55 \mathrm{eV}$ near-infrared pulse $(800 \mathrm{~nm}, 3$ $\mathrm{mJ}, 35 \mathrm{fs}$ ) was utilized to pump the $\mathrm{ZnTe}$ crystal to generate $\mathrm{THz}$ pulses. The THz pulses were focused on the sample or reference with a parabolic mirror. The electric field of the $\mathrm{THz}$ pulses was polarized perpendicular with the gaps of the SRRs. The transmitted $\mathrm{THz}$ pulses were collimated and focused in the detection $\mathrm{ZnTe}$ crystal, with which the electro optical (EO) sampling based on Pockels effect can detect the electric field strength of the $\mathrm{THz}$ pulses. We can obtain the time domain signal of the sample and reference individually with this scheme. Then, a Fourier transform was performed on the time domain signal to obtain the frequency spectrum of the reference and metamaterial samples. The transmission spectrum of the sample was calculated by normalize the spectrum of metamaterial sample to the reference. A DC voltage power supply was used to drive the comb-drive actuator. By sweeping the applied voltage, the spectra of the metamaterial were measured at different lateral displacements.

The measured transmission spectra of the metamaterial with different lateral displacements are shown in Figure 6 (a). When the lateral displacement is $0 \mu \mathrm{m}$, there are two resonant modes with resonant frequencies of $1.03 \mathrm{THz}$ and $1.23 \mathrm{THz}$. With the increasing lateral shift, the first resonant mode shifts higher frequency (blueshift) while the second mode shifts to lower frequency (redshift). With the lateral displacement up to $20 \mu \mathrm{m}$, the first mode blueshifts by $60 \mathrm{GHz}$ and the second mode redshifts by $50 \mathrm{GHz}$. At the same time, the transmission amplitude at $1.03 \mathrm{THz}$ is tuned from 0.2 to 0.65 , as shown in Figure 6 (b).

\section{DISCUSSION}

The behavior of the BC-SRRs can be described with different theories [18], including coupled mode theory and mutual capacitance and inductance model [19]. We will use the third method to interpret the presented tunable metamaterial.

We simulated the transmission spectra and surface current distributions of the BC-SRRs with different lateral displacements using CST Microwave Studio. In the simulation, unit-cell boundary condition was applied. The spectra agree [Figure 7 (a)] well with the experimental results [Figure 6 (a)]. When the lateral shift is $0 \mu \mathrm{m}$, the surface currents are in the same direction at the first mode and in the opposite direction at the second mode, as shown in Figure 7 (b) and (c). We define the first mode as symmetric mode and the second mode as the anti-symmetric mode. The mutual inductance and capacitance are positive for the symmetric mode but negative for the

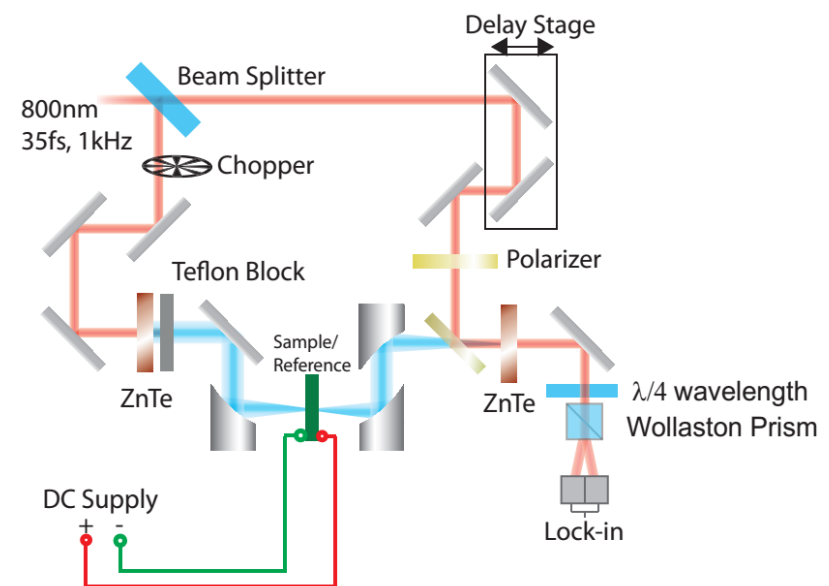

Figure 5: Terahertz time domain spectroscopy for the tunable metamaterial characterization. anti-symmetric mode due to the surface current and charge distribution. With increasing lateral displacement, the absolute value of the mutual inductance and capacitance decrease. As a result, the total inductance and capacitance of the symmetric mode decrease, leading to a blueshift in the resonant frequency; at the same time, the total lumped $\mathrm{L}$ and $\mathrm{C}$ increase, resulting in the redshift of the second mode. When the lateral displacement is about $20 \mu \mathrm{m}$, the two resonators oscillate individually without any coupling as shown in Figure 7 (d) and (e). The simulated transmission spectra agree well with the experimental data and support our qualitative analysis.

\section{CONLCUSION}

We presented our recent process on the dynamically tunable terahertz metamaterial based on broadside-coupled split ring resonators (BC-SRRs). The BC-SRRs are with a symmetric mode and an anti-symmetric mode. With the optimization of the structural design, we achieved $60 \mathrm{GHz}$ redshift of the symmetric mode and 50 $\mathrm{GHz}$ blueshift of the anti-symmetric mode. At the same time, $69 \%$ modulation depth was demonstrated at $1.03 \mathrm{THz}$. The tunable metamaterial has potential applications for dynamic modulation of the $\mathrm{THz}$ radiation and spectral sensing of chemicals.
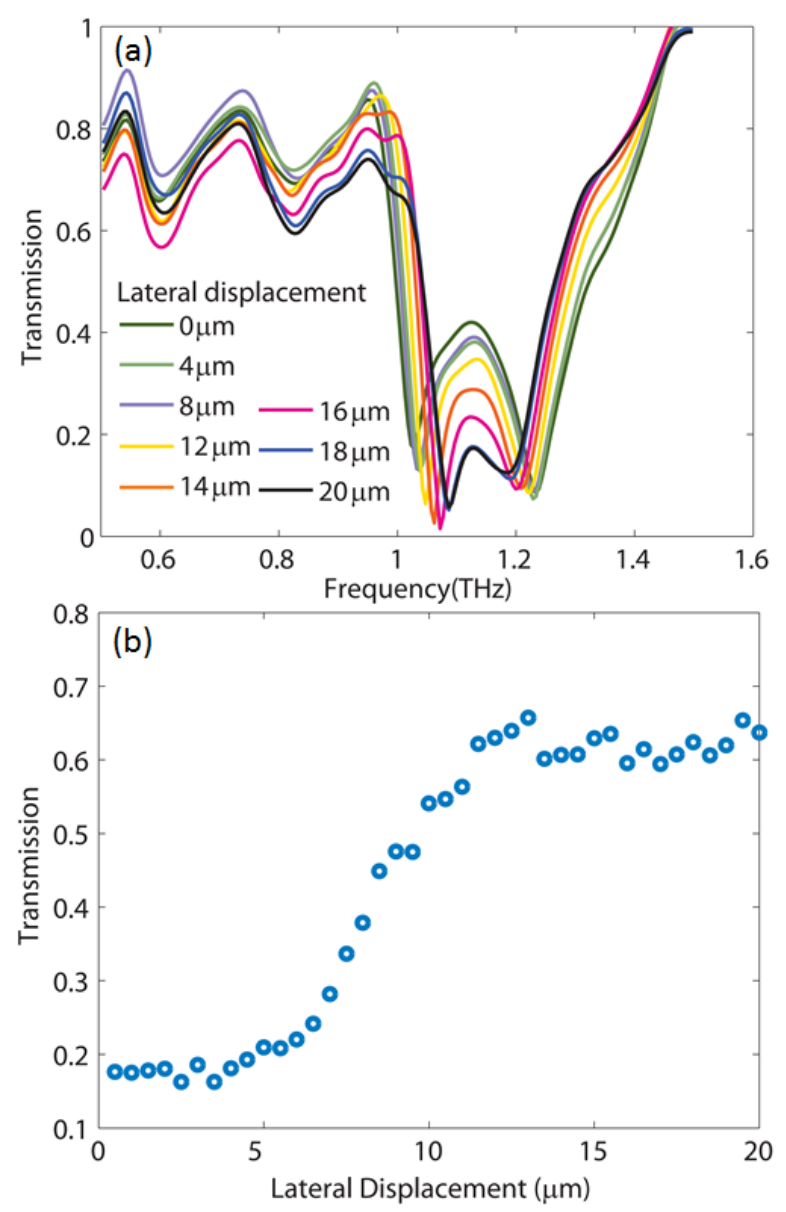

Figure 6: (a) The experimental transmission spectra of the real-time tunable metamaterial. With the increase of the lateral displacement, the two resonance modes merge together due to the decrease of the coupling. (b) The magnitude of transmission at $1.03 \mathrm{THz}$ with different lateral displacement. The transmission is tuned by $69 \%$. 

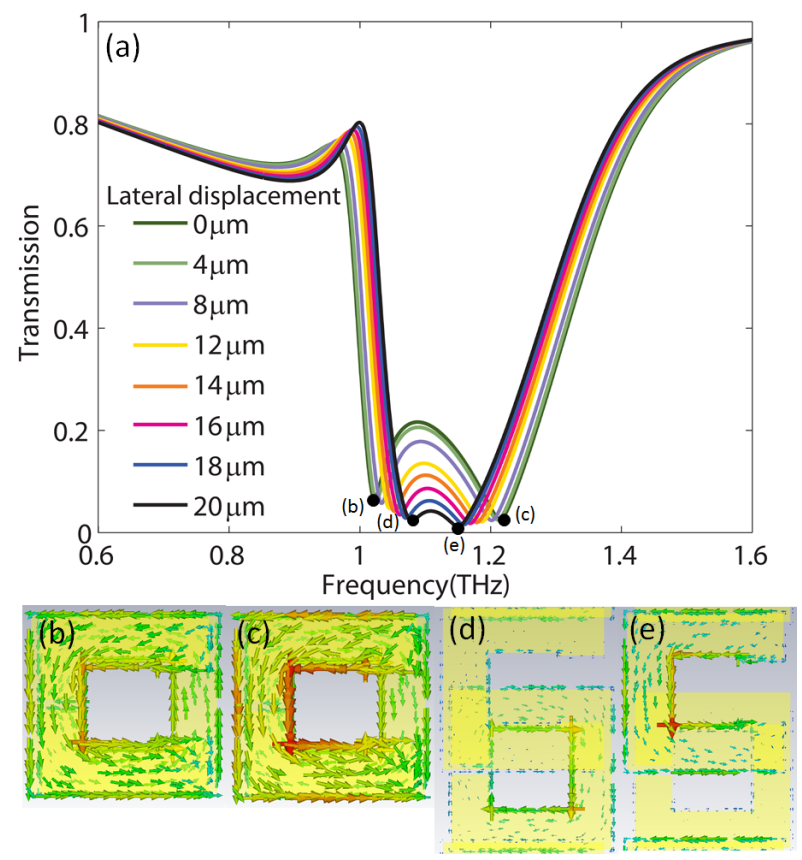

Figure 7: (a) The simulated transmission spectrum at different lateral displacement. (b) - (e) the surface current at the corresponding resonance frequency as shown in (a). Strong coupling exists between the broadside coupled SRRs in (b) and (c), while little coupling in (d) and (e).

\section{ACKNOWLEDGEMENT}

We acknowledge the National Science Foundation (NSF) under Grant No. ECCS-1309835. In addition, we acknowledge support from the Department of Energy (DOE) - Basic Energy Sciences under Grant No. DE-FG02-09ER46643. The authors would like to thank Boston University Photonics Center for technical support.

\section{REFERENCES}

[1] N.I. Zheludev and Y.S. Kivshar, "From Metamaterials to Metadevices", Nature Materials, 11, 917 (2012).

[2] D.R. Smith, J.B. Pendry, and M.C. Wiltshire, "Metamaterials and negative refractive index", Science, 305, 788 (2004).

[3] X. Zhang and Z. Liu, "Superlenses to Overcome the Diffraction Limit", Nature Materials, 7, 435 (2008).

[4] N.I. Landy, S. Sajuyigbe, J.J. Mock, D.R. Smith, and W.J. Padilla, "Perfect Metamaterial Absorber", Physical Review Letters, 100, 207402 (2006).

[5] N. Engheta, "Circuits with Light at Nanoscales: Optical Nanocircuits Inspired by Metamaterials", Science, 317, 1698 (2007).
[6] X. Xu, B. Deng, D. Li, J. Zhang, L. Wong, Q. Zhang, S. Wang, and Q. Xiong, "Flexible Visible-Infrared Metamaterials and Their Applications in Highly Sensitive Chemical and Biological Sensing", Nano Letters, 11, 3232 (2011).

[7] L. Feng, Y. Xu, W.S. Fegadolli, M. Lu, J.E. Oliveira, V.R. Almeida, Y. Chen, and A. Scherer, "Experimental Demonstration of a Unidirectional Reflectionless Parity-time Metamaterial at Optical Frequencies", Nature Materials, 12, 108 (2012).

[8] D. Shrekenhamer, C.M. Watts, J. Montoya, S. Krishna, and W.J. Padilla, "Metamaterial-based Imaing for Potential Security Applications", Proceeding of SPIE, 8632, 863321 (2013).

[10] H.-T. Chen, W.J. Padilla, M.J. Cich, A.K. Azad, R.D. Averitt, and A.J. Taylor, "A metamaterials solid-state terahertz phase modulator", Nature Photon, 3, 148 (2009).

[11] K. Fan, X. Zhao, J. Zhang, K. Geng, G.R. Keiser, H.R. Seren, G.D. Metcalfe, M. Wraback, X. Zhang, and R.D. Averitt, "Optically Tunable Terahertz Metamaterials on Highly Flexible Substrates", IEEE Transcation on Terahertz Science and Technology, 3, 702 (2013).

[12] H. Tao, A.C. Strikwerda, K. Fan, W.J. Padilla, X. Zhang, and R.D. Averitt, "MEMS Based Structurally Tunable Metamaterials at Terahertz Frequencies", Journal of Infrared, Milimeter and Terahertz Waves, 32, 580 (2011).

[13] E. Ekmekci, A.C. Strikwerda, K. Fan, G.R. Keiser, X. Zhang, G. Turhan-Sayan, and R.D. Averitt, "Frequency Tunable Terahertz Metamaterials Using Broadside Coupled Split-ring Resonators", Physical Review B, 83, 193103 (2011).

[14] A.Q. Liu, W.M. Zhu, D.P. Tsai, and N.I. Zheludev, "Micromachined Tunable Metamaterials: A Review", Journal of Optics, 14, 114009 (2011).

[15] A.D. Boardman, V.V. Grimalsky, Y.S. Kivshar, M. Lapine, and N. Litchinister, "Active and Tunable Metamaterials", Laser Photonics Review, 5, 287 (2011).

[16] X. Zhao, K. Fan, G.R. Keiser, H.R. Seren, R.D. Averitt, and X. Zhang, "A Real-Time Tunable Terahertz Metamaterial Based on Broadside-Coupled Split Ring Resonators", Proceeding of the 18th International Conference on Solid-State Sensors, Actuators, and Microsystems, Anchorage, AK, June 21-25, 2015, pp. 315-318.

[17] G.R. Keiser, A.C. Strikwerda, K. Fan, V. Young, X. Zhang, and R.D. Averitt, "Decoupling Crossover in Asymmetric Broadside Coupled Split-Ring Resonators at Terahertz Frequencies", Physical Review B, 88, 023101 (2013).

[18] H. Haus and W.P. Huang, "Couple-Mode Theory", Proceedings of the IEEE, 79, 10 (1991).

[19] G.R, Keiser, K. Fan, X. Zhang, and R.D. Averitt, "Towards Dynamic, Tunable, and Nonlinear Metamaterials via Near Field Interactions: A Review", Journal of Infrared, Milimeter and Terahertz Waves, 34, 709 (2013).

\section{CONTACT}

*X. Zhang, tel: + 1.617.358.2702; xinz@bu.edu 